M. Brodmann and W. Vogel

Nagoya Math. J.

Vol. 131 (1993), 109-126

\title{
BOUNDS FOR THE COHOMOLOGY AND THE CASTELNUOVO REGULARITY OF CERTAIN SURFACES
}

\author{
M. BRODMANN AND W. VOGEL $*)$
}

\section{Introduction}

Let $X \subseteq \mathbf{P}^{r}$ be a reduced, irreducible and non-degenerate projective variety over an algebraically closed field $K$ of characteristic 0 . Let $\operatorname{reg}(X)$ be the Castelnuovo-Mumford regularity of the sheaf of ideals $\mathscr{J} \subseteq \mathscr{O}_{\mathbf{P}^{r}}$ associated to $X$. Then it is an open problem-due to D. Eisenbud (see e.g. [E-Go])-whether

$$
\operatorname{reg}(X) \leq \operatorname{deg}(X)-\operatorname{codim}(X)+1,
$$

where $\operatorname{deg}(X)$ denotes the degree of $X$ and $\operatorname{codim}(X)$ denotes the codimension of $X$. In many cases, this inequality has been proven to hold true.

So Gruson, Lazarsfeld and Peskine have shown (0.1) in case $X$ is a curve (s. [G-L-P]). For smooth surfaces, the requested inequality has been proved by Lazarsfeld [L]. Stückrad and Vogel $\left[\right.$ St $\left.-V_{1}\right]$ have settled the case of an arbitrary Buchsbaum variety $X$. In [Ho-V] the above inequality is shown if $X$ is an arithmetically quasi-Buchsbaum (1-Buchsbaum) surface.

Nowadays, the study of $k$-Buchsbaum varieties $(k \geq 1)$ is of growing importance (see e.g. [Fi-V]). It should be noted that any projective locally Cohen-Macaulay variety is $k$-Buchsbaum for some $k \geq 0$. A systematic way of constructing such varieties is given by the methods of [Ev-Gr] and [Go].

One of the goals of this paper is to study the first case in which the problem $(0.1)$ is open: The case of (possibly singular) surfaces which are 2-Buchsbaum. We namely shall prove that such surfaces satisfy the inequality.

$$
\operatorname{reg}(X) \leq \operatorname{deg}(X)-r+3
$$

If $\operatorname{deg}(X) \leq r$, one may conclude by a theorem found in [Ho-St-V]. If $\operatorname{deg}(X)$

Received January 31, 1992.

*) Partially supported by the SNF, Ges. Nr. 21-27687. 89 
$\geq r+1$ and if the generic hyperplane section $Y$ of $X$ is either non-smooth or non-rational, we shall use a lemma of [Ho-M-V] and the fact that $Y$ is not of maximal regularity (s. [G-L-P]) to conclude. So, the crucial point is to study the case where the generic hyperplane section $Y$ is smooth and rational. In the terminology of $\left[\mathrm{Br}_{2}\right]$ this means that $X$ is normal and of sectional genus 0 .

We study this case in detail, thereby admitting that $X$ is arithmetically $k$-Buchsbaum for some natural number $k$. (If $X$ is normal, such a $k$ always exists). Observe that (if we do not want to specify $k$ ) this case may be characterised by the facts that $X$ is Cohen-Macaulay and has smooth and rational generic hyperplane sections. In this situation one of our main results (see (3.11)) gives bounds for the first local cohomology module of the homogeneous coordinate ring of $X$ in terms of $r, \operatorname{deg}(X)$ and $k$.

Setting $k=2$, these bounds easily furnish the inequality (0.2) if $\operatorname{deg}(X) \geq r+2$ and $\operatorname{deg}(X)>6$. The remaining cases $\operatorname{deg}(X)=r+1$ and $\operatorname{deg}(X)=6, r=4$ need a more detailed analysis, which heavily relies on the assumption that $k$ equals 2 .

Acknowledgement. We would like to thank Lê Tuãn Hoa for his helpful comments on the proof of Proposition (4.1).

\section{Preliminary results}

Let $K$ be an algebraically closed field of characteristic 0 . Let $\mathbf{P}^{\gamma}=$ $\operatorname{Proj}\left(K\left[X_{0}, \ldots, X_{r}\right]\right)$ be the projective space of dimension $r$ over the field $K$. First, we recall the definition of a closed $k$-Buchsbaum scheme in $\mathbf{P}^{r}$.

Let $Y=\operatorname{Proj}(A)$ be a closed subscheme of $\mathbf{P}^{r}$ and let $k \geq 0$ be an integer. Let $P=\mathrm{A}_{>0}$ be the irrelevant ideal of $A$. Then $Y$ is called (arithmetically) $k$-Buchsbaum if the local cohomology modules $H_{P}^{i}(A)$ are annihilated by $P^{k}$ for $i=0, \ldots, \operatorname{dim}(A)-1$.

Let $X \subseteq \mathbf{P}^{r}$ be a closed non-degenerate irreducible and reduced surface. Assume that

(1.1) $X$ is arithmetically $k$-Buchsbaum for some $k \in \mathbf{N}$.

(1.2) If $\mathbf{P}^{r-1}=\mathrm{H} \subseteq \mathbf{P}^{r}$ is a generic hyperplane, the (scheme-theoretic) intersection $X \cap H$ is a smooth (connected) and rational curve. 
(1.3) Remark. Let $Y$ be a pure-dimensional closed subscheme of $\mathbf{P}^{r}$ with ideal sheaf $\mathscr{g} \subseteq \mathscr{O}_{\mathbf{P}}$. We recall that the following conditions are equivalent

(a) $Y$ is a locally Cohen-Macaulay scheme.

(b) There is an integer $k \geq 0$ such that $Y$ is $k$-Buchsbaum.

In terms of Serre cohomology the condition that $Y$ is $k$-Buchsbaum may be expressed as follows:

(c) $\left(X_{0}, \ldots, X_{r}\right)^{k}\left[\underset{n \in \mathbf{Z}}{\bigoplus} H^{i}\left(\mathbf{P}^{r}, \mathscr{g}(n)\right)\right]=0$ for $i=1, \ldots, \operatorname{dim}(X)$.

\section{(1.4) Proposition. $X$ is normal.}

Proof. By assumption (1.1), $X$ is a CM-surface. It thus remains to be shown, that $X$ has only finitely many singular points. Let us assume that this is not the case ! Then, the singular locus $Y$ of $X$ is a closed one-dimensional subset of $X$. Now, let $H \subseteq \mathbf{P}^{r}$ be a generic hyperplane. By (1.2) the scheme-theoretic intersection $X \cap H$ is a smooth curve. Hence the points of $X \cap H$ are regular points of $X$. This implies that $Y \cap H=\emptyset$ and thus contradicts our assumption that $Y$ is of dimension 1 .

Let $A$ be the homogeneous coordinate ring of our surface $X$. So $A$ is a graded integral $K$-algebra of Krull dimension 3, which is generated over $K$ by finitely many forms of degree one. We write $A_{n}$ for the $n$-th homogeneous part of $A$. The dimension of the $K$-vector-space $A_{n}$ will be denoted by $a_{n}$. Moreover we write $H^{i}$ for the $i$-th local cohomology functor $H_{P}^{i}: \operatorname{Mod}_{A}^{+} \rightarrow \operatorname{Mod}_{A}^{+}$defined on the category $\operatorname{Mod}_{A}^{+}$of graded $A$-modules by the irrelevant ideal $P=A_{>0}$ of $A$. By $H^{i}(\cdot)_{n}$ we denote the $n$-th homogeneous part of the functor $H^{i},(n \in \mathbf{Z})$. If $M$ is a finitely generated graded $A$-module, we write $h^{i}(M)_{n}$ for the (finite) dimension of the $K$-vector-space $H^{i}(M)_{n}$.

Now, we may formulate the following result on the local cohomology modules $H^{i}(A)$ of $A$ :

(1.5) Proposition. a) $H^{0}(A)=0$.

b) $H^{1}(A)_{n}=0$, for all $n \leq 0$.

c) $H^{2}(A)=0$.

d) $H^{3}(A)_{n}=0$, for all $n \geq-1$. 
Proof. a) follows from the fact that $A$ is integral and of positive dimension, b) from the reducedness and connectivity of $X$.

To prove c) and d) we choose a generic element $h \in A_{1}-\{0\}$ such that $Y:=\operatorname{Proj}(A / h A)$ is a smooth, connected and rational curve. (Such elements $h$ exist by our hypothesis (1.2)). Applying cohomology to the sequence $0 \rightarrow A$ $\stackrel{h}{\rightarrow} A(1) \rightarrow A / h A(1) \rightarrow 0$ we get the following exact sequences

$$
\begin{aligned}
0 & \rightarrow H^{0}(A / h A)_{n+1} \rightarrow H^{1}(A)_{n} \stackrel{h}{\rightarrow} H^{1}(A)_{n+1} \rightarrow \\
& \rightarrow H^{1}(A / h A)_{n+1} \rightarrow H^{2}(A)_{n} \stackrel{h}{\rightarrow} H^{2}(A)_{n+1} \rightarrow \\
& \rightarrow H^{2}(A / h A)_{n+1} \rightarrow H^{3}(A)_{n} \stackrel{h}{\rightarrow} H^{3}(A)_{n+1} \rightarrow 0 .
\end{aligned}
$$

As $Y$ is smooth and rational its geometric genus vanishes, and hence we have

$$
H^{2}(A / h A)_{n+1}=H^{1}\left(Y, \mathscr{O}_{Y}(n+1)\right)=0
$$

for all $n \geq-1$. So, for all $n \geq-1$ we obtain isomorphisms $H^{3}(A)_{n} \cong H^{3}(A)_{n+1}$. As $H^{3}(A)_{n}=0$ for $n \gg 0$, this implies d).

For all $n \geq-1$ the vanishing of $H^{2}(A / h A)_{n+1}$ implies epimorphisms $H^{2}(A)_{n} \stackrel{h}{\rightarrow} H^{2}(A)_{n+1} \rightarrow 0$. As $X$ is normal (s. (1.4)) the vanishing theorem of Kodaira-Mumford-Ramanujam $\left[\mathrm{Mu}_{1}\right]$ furnishes that $H^{2}(A)_{n}=H^{1}\left(Y, \mathscr{O}_{X}(n)\right)=0$ for all $n \leq-1$. Altogether, this proves c).

If we use the terminology of $[\mathrm{Fi}-\mathrm{V}]$ or $[\mathrm{Ho}-\mathrm{M}-\mathrm{V}]$ the next corollary shows that $X$ is $(k, 2)$-Buchsbaum.

(1.6) Corollary. Let $l \in A_{1} \backslash\{0\}$. Then $\operatorname{Proj}(A / l A)$ is again $k-B u c h s b a u m$.

Proof. By (1.5) $H^{2}(A)=H^{0}(A)=0$. Hence (1.6) follows from the exact sequence of the previous proof applied with $l$ instead of $h$.

(1.7) Remarks. A) As an immediate consequence of (1.5) we get exact sequences

$$
0 \rightarrow H^{0}(A / l A)_{n+1} \rightarrow H^{1}(A)_{n} \stackrel{l}{\rightarrow} H^{1}(A)_{n+1} \rightarrow H^{1}(A / l A)_{n+1} \rightarrow 0
$$

for all $n \in \mathbf{Z}$ and all $l \in A_{1}-\{0\}$.

B) As another consequence of (1.5) we obtain

$$
H^{2}(A / l A)_{n}=0 \text {, for all } n \in \mathbf{N}_{0} \text {, and all } l \in A_{1}-\{0\} .
$$

We write $d$ for the degree of $X$. Then we have 
(1.8) Lemma. Let $l \in A_{1}-\{0\}$. Then $h^{1}(A)_{1}=h^{1}(A / l A)_{1}=d-r+1$.

Proof. By (1.5) b) and the sequences of (1.7) A) we get $h^{1}(A)_{1}=$ $h^{1}(A / l A)_{1}$. By (1.2) we may choose $l \in A_{1}-\{0\}$ such that $Y=\operatorname{Proj}(A / l A)$ is a smooth connected rational curve. It suffices to prove that $h^{1}(A / l A)_{1}=d-r+1$ for such a particular element $l$. The homogeneous coordinate ring $B:=(A / l A) /$ $H^{0}(A / l A)$ of $Y$ satisfies $H^{1}(B)=H^{1}(A / l A)$. It thus remains to be shown that $h^{1}(B)_{1}=d+r-1$.

To do so, we write $b_{n}$ for the dimension of the $K$-vector space $B_{n}$ of all $n$-forms of $B$. As $Y$ is a smooth, rational and connected curve of degree $d$, its characteristic polynomial $\chi_{Y}$ satisfies the equation $\chi_{Y}(n)=d n+1=h^{0}(Y$, $\left.\mathfrak{O}_{Y}(n)\right)=b_{n}+h^{1}(B)_{n}$ for all $n \geq 0$. As $Y$ is non-degeneratedly embedded into the generic hyperplane by which it is cut out $[F-V]$, we have $b_{1}=r$. Consequently we obtain $d+1=\chi_{Y}(1)=h^{1}(B)+r$, hence our claim.

(1.9) Proposition. The characteristic polynomial $\chi_{X}$ of $X$ is given by

$$
\chi_{X}(T)=\frac{d}{2} T^{2}+\frac{d+2}{2} T+1
$$

Proof. In view of (1.5) we have $\chi_{X}(n)=a_{n}+h^{1}(A)_{n}$ for all $n \geq 0$. As $X$ is of degree $d$, we also have $\chi_{X}(T)=\frac{d}{2} T^{2}+b T+c$ with $b, c \in \mathbf{Q}$. Putting $T=0$ we get $c=\chi_{X}(0)=a_{0}+h^{1}(A)_{0}=1$. Putting $T=1$ we get $\frac{d}{2}+b+1=$ $\chi_{X}(1)=a_{1}+h^{1}(A)_{1}$. As $X$ is non-degenerate, we have $a_{1}=r+1$. By (1.8) $h^{1}(A)_{1}=d-r+1$. Therefore we get $\frac{d}{2}+b+1=d+2$, hence $b=\frac{d+2}{2}$.

\section{Generic pairs of linear forms}

We keep the notations and hypotheses of the previous section. We say that two elements $h, l \in A_{1}$ are a generic pair of linear forms if the following conditions are satisfied:

(2.1) $Y:=\operatorname{Proj}(A / h A)$ is a smooth, connected and rational curve.

(2.2) $Z:=\operatorname{Proj}(A /(h A+l A))$ is reduced and consists of $d:=\operatorname{deg}(X)$ points which are in general position in the subspace $\mathbf{P}^{r-2}$ of $\mathbf{P}^{r}$ which is defined by $h$ and $l$. 
(2.3) Remarks. A) We recall that $d$ points are said to be in general position in $\mathbf{P}^{r-2}$ if no hyperplane $H^{\prime}$ of $\mathbf{P}^{r-2}$ contains $r-1$ of these points.

B) In view of our assumption (1.2) there is a linear form $h \in A_{1}$ which satisfies (2.1). If we fix such an $h$, the general position lemma [H] shows that there is a linear form $l \in A_{1}$ for which (2.2) is satisfied. Hence generic pairs of linear forms exist.

The following lemma will play an important role. It is an immediate consequence of $\left[\mathrm{St}-\mathrm{V}_{2}\right.$, Thm. 1], (see also [N, 2.1.2.2]).

(2.4) Lemma. Let $h, l \in A_{1}$ be a generic pair of linear forms and let $C:=$ $A /(h A+l A)$. Then, $h^{1}(C)_{n} \leq \max \{0, d-1-n(r-2)\}$ for all $n \geq 0$.

Now, we write

$$
t:=\frac{d-1}{r-2}, \quad(d=\operatorname{deg}(X))
$$

Using this notation, we have the following result, which follows immediately from (2.4).

(2.6) Lemma. Let $h, l \in A_{1}$ be a generic pair of linear forms and let $C:=$ $A /(h A+l A)$. Then $H^{1}(C)_{n}=0$ for all $n \geq t$.

Now, we fix a generic pair $h, l \in A_{1}$ and study the functions $n \mapsto$ $h^{1}(A /(\lambda h+\mu l) A)_{n}$ for arbitrary pairs $(\lambda, \mu) \in K^{2}-\{(0,0)\}$ of coefficients. To do so, we recall some useful facts on cohomological Hilbert functions and Castelnuovo-Mumford regularities.

(2.7) Remarks. A) Let $s \geq 2$ be an integer and let $S$ be the polynomial ring $K\left[X_{0}, \ldots, X_{s}\right]$. Write $\mathbf{P}^{s}=\operatorname{Proj}(S)$. Let $T$ be a graded homomorphic image of $S$ and consider the closed subscheme $W=\operatorname{Proj}(T)$ of $\mathbf{P}^{s}$ defined by $T$. Let $g_{W} \subseteq$ $\mathcal{O}_{\mathbf{P} s}$ be the ideal sheaf associated to $W$. Then, the Serre-Grothendieck correspondence yields graded isomorphisms

$$
H^{i}(T) \cong \bigoplus_{n \in \mathbf{Z}} H^{i}\left(\mathbf{P}^{S}, g_{W}(n)\right) ;(i=1, \ldots, s-1),
$$

which give rise to the equations

$$
h^{i}(T)_{n}=h^{i}\left(\mathbf{P}^{S}, \mathscr{g}_{W}(n)\right\} ;(i=1, \ldots, s-1 ; n \in \mathbf{Z}) .
$$


B) Let $m \in \mathbf{N}_{0}$. Then $\mathscr{g}_{W}$ is $m$-regular in the sense of Castelnuovo and Mumford iff $h^{i}\left(\mathbf{P}^{S}, g_{W}(n)\right)=0$ for all $n \geq m-i$ and all $i \geq 1$. So, the $m$-regularity of $g_{W}$ is equivalent to the statement that $h^{i}(T)_{n}=0$ for all $n \geq m-i$ and all $i \in\{1, \ldots, \operatorname{dim}(W)+1\}$.

Now, we are ready to prove the following auxiliary result:

(2.8) Lemma. Let $h, l \in A_{1}$ be a generic pair, let $(\lambda, \mu) \in K^{2}-\{(0,0)\}$ and let $B=A /(\lambda h+\mu l) A$. Then $h^{1}(B)_{n} \leq \max \left\{0, h^{1}(B)_{n-1}-1\right\}$ for all $n \geq t+1$.

Proof. There is a linear form $u \in K h+K l \subseteq A_{1}$ such that $(\lambda h+\mu l) A+$ $u A=h A+l A$. We write $\mathbf{P}^{r-1}$ for the hyperplane defined by $\lambda h+\mu l$ in $\mathbf{P}^{r}$ and consider $Y:=\operatorname{Proj}(B)$ as a closed subscheme of $\mathbf{P}^{r-1}$. The linear form $u$ defines a hyperplane $L$ in $\mathbf{P}^{\gamma-1}$ whose scheme-theoretic intersection with $Y$ is given by $Z:=\operatorname{Proj}(A /(h A+l A))$. Let $\mathscr{g}_{Z} \subseteq \mathscr{O}_{L}$ be the ideal sheaf associated to $Z$.

In view of $(2.7) \mathrm{B}$ ) we get from (2.6) that $\mathscr{g}_{Z}$ is $n$-regular for all $n \geq t+1$. Now, let $\mathscr{J}_{Y} \subseteq \mathscr{O}_{\mathbf{P}^{r-1}}$ be the ideal sheaf associated to $Y$. As $X$ is a CM-scheme, so is $Y$. So, the members of $\operatorname{Ass}\left(\mathscr{O}_{Y}\right)$ are all generic and thus avoided by $L$. As a consequence we have $\mathscr{g}_{Z}=\mathscr{g}_{Y} \mid L$. Now, the Lemma of Mumford-Le Poitier $\left[\mathrm{Mu}_{2}\right.$, p. 102] shows that $\left.h^{1}\left(\mathbf{P}^{r-1}, \mathscr{g}_{Y}(n)\right) \leq \max \left\{0, h^{1}(n-1)\right)-1\right\}$ for all $n \geq t+1$. In view of (2.7) A) this proves our claim.

As a complement to (2.8) we have

(2.9) Lemma. Let $B$ be as in (2.8) and assume that $n-1<t \leq n$. Then $h^{1}(B)_{n} \leq h^{1}(B)_{n-1}$.

Proof. We write $C=A /(h A+l A)$ and choose $u \in A_{1}$ as in the previous proof. Writing $I$ for the kernel of the multiplication map $u: B \rightarrow B$ we get a graded exact sequence $0 \rightarrow B / I(-1) \stackrel{u}{\rightarrow} B \rightarrow C \rightarrow 0$. As $I \subseteq H^{0}(B)$ we have $H^{1}(B / I)=H^{1}(B)$. So, applying cohomology we get exact sequences

$$
H^{1}(B)_{n-1} \stackrel{u}{\rightarrow} H^{1}(B)_{n} \rightarrow H^{1}(C)_{n},(h \in \mathbf{Z}) .
$$

Now, we conclude by (2.6).

(2.10) Lemma. Let $h, l \in A_{1}$ be a generic pair of linear forms. Then

a) $H^{1}(A / h A)_{n}=0$ for all $n \geq d-r+2$.

b) $H^{1}(A /(\lambda h+\mu l) A)_{n}=0$ for all $n \geq t+k-1$ and for all pairs $(\lambda, \mu) \in$ 
$K^{2}-\{(0,0)\}$

Proof. a) Let $H=\mathbf{P}^{r-1} \subseteq \mathbf{P}^{r}$ be the hyperplane defined by $h$. Then, $Y:=\operatorname{Proj}(A / h A)$ is a smooth, connected and non degenerate curve in $H$. Let $\mathscr{g}_{Y} \subseteq \mathscr{O}_{H}$ the ideal sheaf associated to $Y$. Then, by [G-L-P] the sheaf $\mathscr{g}_{Y}$ is $(d-r$ $+3)$-regular. So (2.7) B) proves our claim.

b) We use the notations of (2.9). Lemma (2.6) and the sequences $(*)$ of the proof of (2.9) give rise to epimorphisms $u^{k}: H^{1}(B)_{n-k} \rightarrow H^{1}(B)_{n}$ for all $n \geq t+$ $k-1$. By (1.6), these epimorphisms are all 0 . So $H^{1}(B)_{n}$ vanishes for all $n \geq t+$ $k-1$.

Next, we define the integer $\alpha$ by

$$
\alpha<t \leq \alpha+1
$$

and the function $F: \mathbf{N}_{o} \rightarrow \mathbf{Z}$ by

$$
F(n):=(d-1) n+(2-r)\left(\begin{array}{c}
n+1 \\
2
\end{array}\right)
$$

Thereby we use the convention that $\left(\begin{array}{l}1 \\ 2\end{array}\right)=0$.

Using these notations we get the following result:

(2.13) Proposition. Let $h, l \in A_{1}$ be a generic pair of linear forms. Let $(\lambda, \mu) \in K^{2}-\{(0,0)\}$. Then:

$$
h^{1}(A /(\lambda h+\mu 1) A)_{n} \leq \begin{cases}F(n) & ; 0 \leq n \leq \alpha . \\ L(n):=\max \{0, F(\alpha)-(n-\alpha-1)\} & ; \alpha<n .\end{cases}
$$

Proof. In view of (2.8) and (2.9) it remains to be shown that $h^{1}(B)_{n}$ $\leq F(n)$ for $0 \leq n \leq \alpha$, where $B$ stands for $A /(\lambda h+\mu l) A$. This is done by induction on $n$. If $n=0$, the sequence $H^{1}(A)_{o} \rightarrow H^{1}(B)_{o} \rightarrow H^{2}(A)_{-1}$ together with (1.5) shows that $h(B)_{o}=0 \leq F(0)$. So, let $0<n \leq \alpha$. The sequence (*) of (2.9) together with (2.4) furnishes the inequality $h^{1}(B)_{n} \leq h^{1}(B)_{n-1}+d-1-$ $(r-2) n$. Applying the hypothesis of induction we finally obtain $h^{1}(B)_{n} \leq F(n-$ 1) $+d-1-(r-2) n=F(n)$.

\section{Bounding the numbers $h^{1}(A)_{n}$}

We keep the previous notations and hypotheses. The aim of this section is to 
give bounds on the numbers $h^{1}(A)_{n}$. We start with the following auxiliary result:

(3.1) Lemma a) $h^{1}(A)_{d-r+2} \leq h^{1}(A)_{d-r+1}$.

b) $h^{1}(A)_{n} \leq \max \left\{0, h^{1}(A)_{n-1}-1\right\}$ for all $n \geq d-r+3$.

Proof. Let $h \in A_{1}$ be a generic linear form. The inequality a) now follows immediately from the exact sequence $H^{1}(A)_{d-r+1} \stackrel{h}{\rightarrow} H^{1}(A)_{d-r+2} \rightarrow H^{1}(A / h A)_{d-r+2}$ and from (2.10) a). To prove statement b), let $H=\mathbf{P}^{r-1} \subseteq \mathbf{P}^{r}$ be the hyperplane $h$ defines in $\mathbf{P}^{r}$. Then, $Y:=\operatorname{Proj}(A / h A)$ is a smooth, connected and non-degenerate curve in $H$. Let $\mathscr{g}_{Y} \subseteq \mathcal{O}_{\mathbf{P}^{r-1}}$ the ideal sheaf associated to $Y$. Then $\mathscr{g}_{Y}$ is $(d-r+$ 3)-regular by [G-L-P]. Let $\mathscr{g}_{X} \subseteq \mathcal{O}_{\mathbf{P}^{r}}$ be the vanishing ideal sheaf of $X$. Then $\mathscr{g}_{Y}=\left.\mathscr{g}_{X}\right|_{H}$ allows to apply $\left[\mathrm{Mu}_{2}, \mathrm{p} .102\right]$, hence to conclude that $h^{1}(A)_{n}$ $\leq \max \left\{0, h^{1}(A)_{n-1}-1\right\}$ (s. (2.7) A)).

As a first application of this we get the following result, in which $\alpha, F(n)$, $L(n)$ are defined respectively by $(2.11,12,13)$, and in which

$$
\begin{gathered}
G(n):=(d-1)\left(\begin{array}{c}
n+1 \\
2
\end{array}\right)+(2-r)\left(\begin{array}{c}
n+2 \\
3
\end{array}\right),\left(n \in \mathbf{N}_{o}\right), \\
r:=d-r+2 .
\end{gathered}
$$

(3.5) Proposition.

$$
h^{1}(A)_{n} \leq \begin{cases}G(n) & ; 0 \leq n \leq \alpha . \\ M(n):=G(\alpha)+\sum_{m=\alpha+1}^{n} L(m) & ; \alpha<n \leq \gamma-1 . \\ M(\gamma-1) & ; n=\gamma . \\ \max \{0, M(\gamma)-(n-\gamma)\} & ; n>\gamma .\end{cases}
$$

Proof. Let $h \in A_{1}$ be a generic element. Then, the sequences $H^{1}(A)_{n-1}$ $\stackrel{h}{\rightarrow} H^{1}(A)_{n} \rightarrow H^{1}(A / h A)_{n}$ together with (1.5) b) show that

$$
\begin{aligned}
& h^{1}(A)_{n} \leq \sum_{m=0}^{n} h^{1}(A / h A)_{m} \text { for } 0 \leq n \leq \alpha, \\
& h^{1}(A)_{n} \leq h^{1}(A)_{\alpha}+\sum_{m=\alpha+1}^{n} h^{1}(A / h A)_{m} \text { for } \alpha<n<\gamma .
\end{aligned}
$$

As $G(n)=\sum_{m=0}^{n} F(m)$ we get the requested estimate in the range $0 \leq n \leq \gamma-1$. Now, the cases $n \geq \gamma$ are clear from (3.1). 
(3.6) COROLlary. a) $h^{1}(A)_{n}=0$ for all $n \geq M(\gamma)+\gamma$.

b) $k \leq M(\gamma)+\gamma-1$.

(3.7) Example. Let $d=r+1$. Then obviously $\alpha=1, \gamma=3$. By (3.5) the values $h^{1}(A)_{n}$ are bounded as follows:

\begin{tabular}{c|c|c|c|c|c|c|c|c|c|}
$n$ & 0 & 1 & 2 & 3 & 4 & 5 & 6 & 7 & $\cdots$ \\
\hline$h^{1}(A)_{n} \leq$ & 0 & 2 & 4 & 4 & 3 & 2 & 1 & 0 & $\cdots$
\end{tabular}

In particular we get $k \leq 6$.

For the previous bounds it is not necessary to know $k$. If we know $k$, sharper bounds may be deduced. To obtain them, we need an auxiliary result. To state it, we introduce the invariant

$$
\beta_{o}:=\alpha+\min \{k, F(\alpha)+1\} .
$$

(3.9) Lemma. $h^{1}(A)_{n} \leq \max \left\{0, h^{1}(A)_{n-1}-1\right\}$ for all $n \geq \beta_{o}$

Proof. Let $h, l \in A_{1}$ be a generic pair of linear forms. Then, for any pair $(\lambda, \mu) \in K^{2}-\{(0,0)\}$ we have exact sequences $H^{1}(A)_{n-1} \stackrel{\lambda h+\mu l}{\longrightarrow} H^{1}(A)_{n} \rightarrow$ $H^{1}(A /(\lambda h+\mu l) A)_{n}$. If $n \geq \beta_{o}$ the last term in the above sequence vanishes by $(2.10)$ b) or by (2.13). So we get epimorphisms $(\lambda h+\mu l): H^{1}(A)_{n-1} \rightarrow H^{1}(A)_{n} \rightarrow$ 0 . Now, we may conclude by $\left[\mathrm{Br}_{1},(3.2)\right]$.

Next, we set
a) $\beta:=\min \left\{\beta_{o}, \gamma\right\}$,
b) $\varepsilon:=\left\{\begin{array}{l}0, \text { if } \beta_{o}<\gamma \\ 1, \text { if } \beta_{o} \geq \gamma\end{array}\right.$

where $\beta_{o}$ and $\gamma$ are defined according to (3.8) respectively according to (3.4). Now, combining (3.5) and (3.9) we get the following estimate:

(3.11) THEOREM.

$$
h^{1}(A)_{n} \leq \begin{cases}G(n) & ; 0 \leq n \leq \alpha . \\ M(n) & ; \alpha<n \leq \beta-\varepsilon . \\ M(\beta-\varepsilon) & ; n=\beta . \\ \max \{0, M(\beta)-(n-\beta)\} & ; n>\beta .\end{cases}
$$


Now, we give some right-vanishing bounds for the function $n \mapsto h^{1}(A)_{n}$. First of all we have

(3.12) Lemma. $H^{1}(A)_{n}=0$ for all $n \geq \beta+k-1$.

Proof. Let $h \in A^{1}$ be a generic linear form. Then the exact sequences $H^{1}(A)_{m-1} \stackrel{h}{\rightarrow} H^{1}(A)_{m} \rightarrow H^{1}(A / h A)_{m}$ together with (2.10) and (2.13) give rise to epimorphisms $h: H^{1}(A)_{m-1} \rightarrow H^{1}(A)_{m} \rightarrow 0$ for all $m \geq \beta$. So, if $n \geq \beta+k-1$, we get an epimorphism $h^{k}: H^{1}(A)_{n-k} \rightarrow H^{1}(A)_{n} \rightarrow 0$, which at the same time is 0 . Hence $H^{1}(A)_{n}=0$ for all $n \geq \beta+k-1$.

The previous result tells us in particular that $H^{1}(A)_{n}$ vanishes whenever $n \geq \alpha+F(\alpha)+k$ or $n \geq \alpha+2 k-1$. We now prove a result which partially improves these estimates.

(3.13) PROPOSITION.

a) $k<F(\alpha)+1 \Rightarrow H^{1}(A)_{n}=0$ for all $n \geq \alpha+F(\alpha)+1$.

b) $k<F(\alpha)+1 \Rightarrow H^{1}(A)_{n}=0$ for all $n \geq \alpha+F(\alpha)+k-1$.

Proof. Let $h, l \in A_{1}$ be a generic pair of linear forms. Then the exact sequence $H^{1}(A)_{\beta_{0}-2} \stackrel{h}{\rightarrow} H^{1}(A)_{\beta_{0}-1} \rightarrow H^{1}(A / h A)_{B_{0}-1}$ together with $h H^{1}(A)_{\beta_{0}-2} \subseteq$ $A_{1} H^{1}(A)_{\beta_{o}-2}$ shows that $W_{\beta_{o}-1}:=H^{1}(A)_{\beta_{o}-1} / A_{1} H^{1}(A)_{\beta_{o}-2}$ is a $K$-vectorspace of dimension $w_{\beta_{0}-1} \leq h^{1}(A / h A)_{\beta_{0}-1}$.

Let $m \geq \beta_{o}$, let $W_{m}:=H^{1}(A)_{m} / A_{m+2-\beta_{o}} H^{1}(A)_{\beta_{0}-2}$ and let $w_{m}$ denote the $K$-dimension of $W_{m}$. Let $\bar{h}, \bar{l}: W_{m-1} \rightarrow W_{m}$ be the linear maps induced respectively by the multiplication maps $h, l: H^{1}(A)_{m-1} \rightarrow H^{1}(A)_{m}$. Let $(\lambda, \mu) \in K^{2}-$ $\{(0,0)\}$. As $m \geq \beta_{o}$, we know already from the proof of (3.9) that $\lambda h+\mu l$ : $H^{1}(A)_{m-1} \rightarrow H^{1}(A)_{m}$ is an epimorphism. Hence the same holds true for the map $\lambda \bar{h}+\mu \bar{l}: W_{m-1} \rightarrow W_{m}$. In view of $\left[\mathrm{Br}_{2},(3.1)\right]$ this shows that $w_{m} \leq \max \left\{0, w_{m-1}\right.$ $-1\}$.

So we get $W_{n}=0$, hence $H^{1}(A)_{n}=A_{n+2-\beta_{o}} H^{1}(A)_{\beta_{0}-2}$ for all $n \geq \beta_{o}-1+$ $w_{\beta_{0}-1}$, thus for all $n \geq \beta_{o}-1+h^{1}(A / h A)_{\beta_{0}-1}$.

Now, let $k<F(\alpha)+1$. Then $\beta_{o}=\alpha+k$ and by (2.13) we get $h^{1}(A / h A)_{\beta_{0}-1}$ $\leq F(\alpha)-(\alpha+k-1-\alpha-1)=F(\alpha)-k+2$. Consequently

$$
H^{1}(A)_{n}=A_{n+2-\alpha-k} H^{1}(A)_{\alpha+k-2}
$$

for all $n \geq \alpha+k-1+F(\alpha)-k+2=\alpha+F(\alpha)+1$. 
If $n \geq \alpha+F(\alpha)+1$, we have $n+2-\alpha-k \geq F(\alpha)+3>k$, and thus get $H^{1}(A)_{n}=A_{n+2-\alpha-k} H^{1}(A)_{\alpha+k-2}=0$. This proves statement a).

To prove statement b), let $k \geq F(\alpha)+1$. Then $\beta_{o}=\alpha+F(\alpha)+1$ shows that $h^{1}(A / h A)_{\beta_{0}-1}=h^{1}(A / h A)_{\alpha+F(\alpha)} \leq 1$ (s. (2.12)). Consequently $H^{1}(A)_{n}=$ $A_{n+1-\alpha-F(\alpha)} H^{1}(A)_{\alpha+F(\alpha)-1}$ for all $n \geq \alpha+F(\alpha)+1$. For $n \geq \alpha+F(\alpha)+k-1$ we have $n+1-\alpha-F(\alpha) \geq k$ and thus obtain $H^{1}(A)_{n}=A_{n+1-\alpha-F(\alpha)}$ $H^{1}(A)_{\alpha+F(\alpha)-1}=0$.

(3.14) Example. Let $d=r+1$. Then, using (3.7), (3.11), (3.12) and (3.13) the numbers $h^{1}(A)_{n}$ may be bounded as shown in the following table

\begin{tabular}{|c|c|c|c|c|c|c|c|c|c|}
\hline$n$ & $\leq 0$ & 1 & 2 & 3 & 4 & 5 & 6 & $\geq 7$ & values of $k$ \\
\hline & 0 & 2 & 4 & 4 & 3 & 2 & 1 & 0 & 5,6 \\
$h^{1}(A)_{n} \leq$ & 0 & 2 & 4 & 4 & 3 & 2 & 0 & 0 & 4 \\
& 0 & 2 & 4 & 4 & 3 & 0 & 0 & 0 & 3 \\
& 0 & 2 & 4 & 3 & 0 & 0 & 0 & 0 & 2 \\
& 0 & 2 & 0 & 0 & 0 & 0 & 0 & 0 & 1 \\
\hline
\end{tabular}

In the cases $k=1,2$ we made use of (3.12) (and (3.11)), whereas in the cases $k=3,4$ we used (3.13). In the next section we shall see that an improvement of these bounds is possible if $k=2$. We namely will show that $h^{1}(A)_{3}=0$ in this case.

\section{The case $k=2$}

The goal of this section is to give regularity bounds for projective surfaces which are 2-Buchsbaum. It turns out, that the case in which (1.2) is satisfied plays a crucial role. Among the surfaces occuring in this case, those which satisfy $\operatorname{deg}(X)=r+1$ are of particular importance. For the moment we therefore keep the notations and hypothesis of the previous section and give the announced improvement of the bounds presented in (3.14).

(4.1) Proposition. Let $d=r+1, r>3, k=2$. Then $H^{1}(A)_{n}=0$ for all $n \geq 3$.

Proof. In view of (3.14) it suffices to show that $H^{1}(A)_{3}=0$. Let us assume to the contrary, that $H^{1}(A)_{3} \neq 0$. 
Let $h, l \in A_{1}$ be a generic pair of linear forms, and let $V$ denote the subspace $A_{1} H^{1}(A)_{1}$ of $H^{2}(A)_{2}$. Let $v$ be the $K$-dimension of $V$. Let $(\lambda, \mu) \in K^{2}-\{(0,0)\}$. As $\beta_{o}=3$ the multiplication map $\lambda h+\mu l: H^{1}(A)_{2} \rightarrow H^{1}(A)_{3}$ is an epimorphism (cf. proof of (3.9)). As $k=2$, this epimorphism maps $V$ to 0 . So, we get induced linear maps $\bar{h}, \bar{l}: H^{1}(A)_{2} / V \rightarrow H^{1}(A)_{3}$ such that $\lambda \bar{h}+\mu \bar{l}: H^{1}(A)_{2} / V \rightarrow H^{1}(A)_{3}$ is an epimorphism for all pairs $(\lambda, \mu) \in K^{2}-\{(0,0)\}$. By $\left[\mathrm{Br}_{1},(3.2)\right]$ this induces that $h^{1}(A)_{2}-v \geq h^{1}(A)_{3}+1 \geq 2$, hence that $h^{1}(A)_{2}-v \geq 2$. As $(\lambda h+$ ul) $H^{1}(A)_{1} \subseteq V$ the exact sequence

$H^{1}(A)_{1} \stackrel{\lambda h+\mu l}{\longrightarrow} H^{1}(A)_{2} \rightarrow H^{1}(A /(\lambda h+\mu l))_{2}$ shows that

$2 \leq h^{1}(A)_{2}-v \leq h^{1}(A)_{2}-\operatorname{dim}_{K}\left[(\lambda h+\mu l) H^{1}(A)_{1}\right] \leq h^{1}(A /(\lambda h+\mu l) A)_{2}=: u$.

As $1<t=\frac{d-1}{r-2}=\frac{r}{r-2} \leq 2$ (2.9) and (1.8) show that $u \leq d-r+1=2$.

So, we have $u=2$, hence $v=\operatorname{dim}_{k}\left[(\lambda h+\mu l) H^{1}(A)_{1}\right]$, thus $V=(\lambda h+$ $\mu l) H^{1}(A)_{1}$ for all $(\lambda, \mu) \in K^{2}-\{(0,0)\}$. Therefore, we have epimorphisms $\lambda h+$ $\mu l: H^{1}(A)_{1} \rightarrow V$ for all non-trivial pairs $(\lambda, \mu) \in K^{2}$. By $\left[\mathrm{Br}_{1},(3.2)\right]$ and (1.8) this furnishes $v \leq h^{1}(A)_{1}-1=1$, (s. (1.8)).

We keep in mind, that the previous arguments have shown that $h^{1}(A /(\lambda h+$ $\mu l) A)_{2}=2$ for any non-trivial pair $(\lambda, \mu) \in K^{2}$. Now, we put $B=A / h A$ and $C=A /(h A+l A)$. Then we write $I$ for the kernel of the multiplication map $B(-1) \stackrel{l}{\rightarrow} B$. As $I \subseteq H^{\circ}(B)$ we have $H^{1}(B)=H^{1}(B / I)$.

So, applying cohomology to the diagram 1 , we get the diagram 2 with exact top row and second column (in which $p$ denotes the map $H^{1}(\pi)$ ).

By (1.5) c) $H^{2}(A)_{o}$ vanishes. By (2.6) we have $H^{1}(C)_{2}=0$. So, we have an epimorphism from $H^{1}(A)_{1}$ to the 2-dimensional space $H^{1}(B)_{2}$ which factors through the space $V$ whose dimension is at most 1 . This contradiction proves our claim.

(4.2) Remark. Let $d=r+1$ and $k=2$. Then, according to (3.14) and (4.1), $h^{1}(A)_{1}=2, h^{1}(A)_{2} \leq 4$, and $h^{1}(A)_{n}=0$ for $n \neq 1,2$ (cf. (1.5) b), (1.8)).

By $\operatorname{reg}(T)$ we denote the Castelnuovo-Mumford regularity of a closed subscheme $T$ of $\mathbf{P}^{r}$. So, if $\mathscr{g}_{T} \subseteq \mathscr{O}_{\mathbf{P}^{r}}$ is the ideal sheaf associated to $T$, we may write

$$
\operatorname{reg}(T)=\inf \left\{m \in \mathbf{Z} \mid H^{i}\left(\mathbf{P}^{r}, \mathscr{g}_{T}(n-i)\right)=0, \text { for all } i>0, \text { for all } n \geq m\right\}
$$

Now, as an application of (4.1) we get 


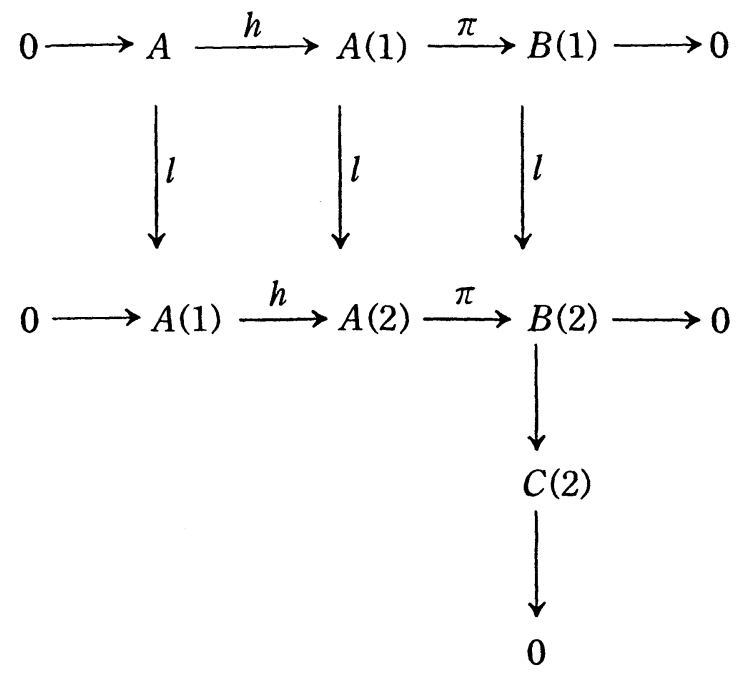

Diagram 1.

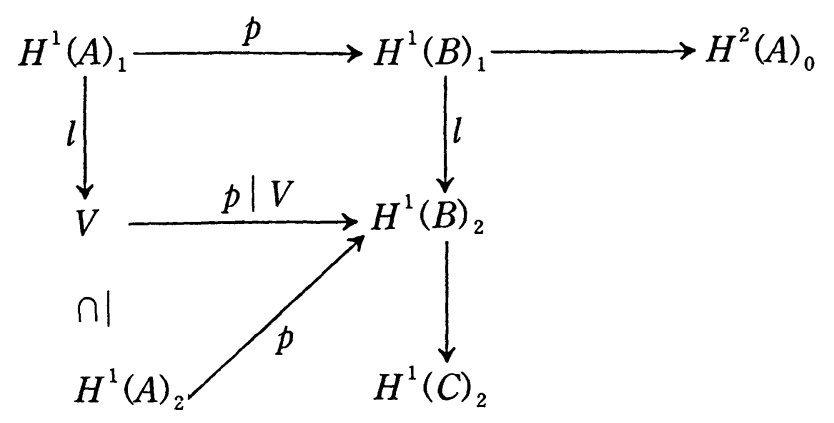

Diagram 2.

(4.4) Proposition. Assume that $X \subseteq \mathbf{P}^{\mathrm{r}}(r>3)$ is a reduced, irreducible non degenerate surface of degree $r+1$. Assume that the hypotheses (1.1) and (1.2) are satisfied. Then

$$
\operatorname{reg}(X) \leq \begin{cases}4, & \text { for } k=2 \\ 3, & \text { for } k=1\end{cases}
$$

Proof. In view of (2.7) B) it suffices to show that $h^{i}(A)_{n}=0$ for all $n \leq 4-$ $i$ (resp. $3-i$ ) for $i=1,2,3$ (according to whether $k=2$ resp. $k=1$ ). So, by 
(1.5) c) it remains to show that $h^{1}(A)_{n}=0$ for all $n \geq 3$ respectively all $n \geq 2$, according to whether $k=2$ or $k=1$. If $k=2$ we may conclude by (4.1). If $k=1$ we use the last row of the table in (3.14).

Now, we want to bound $\operatorname{reg}(X)$ for arbitrary 2-Buchsbaum surfaces. We start with a few auxiliary results

(4.5) Lemma. Let $X \subseteq \mathbf{P}^{r}$ be a closed subscheme of postitive dimension which is arithmetically $k$-Buchsbaum. Then, for a generic hyperplane $H \subseteq \mathbf{P}^{r}$ we have $\operatorname{reg}(X) \leq \operatorname{reg}(X \cap H)+k-1$.

Proof. See [Ho-M-V, (2.5)].

(4.6) Lemma Let $Y \subseteq \mathbf{P}^{3}$ be a reduced, non-degenerate, irreducible curve of degree 6 which is arithmetically 2-Buchsbaum and which satisfies $h^{1}\left(\mathbf{P}^{3}, g_{Y}(3)\right)$ $\neq 1$. Then, $\operatorname{reg}(Y) \leq 4$.

Proof. Assume that $\operatorname{reg}(Y)>4$. Then, according to the table of p. 504 in [G-L-P], the curve $Y$ is smooth, rational, satisfies $h^{1}\left(\mathbf{P}^{3}, \mathscr{g}_{Y}(3)\right)=3$ and is contained in a smooth quadric. We write $B$ for the homogeneous coordinate ring of $Y$. Using our previous notations we then get $h^{1}(B)_{3}=3$, (s. (2.7)).

Now, we apply the general position lemma and find a plane $E=\mathbf{P}^{2} \subseteq \mathbf{P}^{3}$ such that $E \cap Y$ consists of 6 points in general position in $E$. Let $l \in B_{1}$ be the linear form that defines $E$ and put $C=B / l B$. Then $\operatorname{Proj}(C)=E \cap Y$. By our choice of $E$, we get as in the proof of $(2.4)$ that $h^{1}(C)_{n} \leq \max \{0, \operatorname{deg}(Y)-1-$ $n \cdot 2\}=\max \{0,5-2 n\}$. In particular we see that $h^{1}(C)_{2} \leq 1$ and $h^{1}(C)_{3}=0$.

Now, applying cohomology to the short exact sequence $0 \rightarrow B(-1) \stackrel{l}{\rightarrow} B \rightarrow C$ $\rightarrow 0$ and observing that $H^{2}(B)_{n}=0$ for all $n \geq 0$ we get an isomorphism $W:=$ $H^{1}(B)_{2} / l H^{1}(B)_{1} \cong H^{1}(C)_{2}$ and an epimorphism $H^{1}(B)_{2} \stackrel{l}{\rightarrow} H^{1}(B)_{3} \rightarrow 0$. As $B$ is a 2 -Buchsbaum ring, we have $l^{2} H^{1}(B)_{1}=0$. So, the latter map induces an epimorphism $W \rightarrow H^{1}(B)_{3} \rightarrow 0$. Altogether we thus get the contradiction $1 \geq h^{1}(C)_{2}$ $=\operatorname{dim}(W) \geq h^{1}(B)_{3}=3$.

(4.7) Lemma. Let $Y \subseteq \mathbf{P}^{s}(s \geq 3)$ be a reduced, irreducible, non-degenerate curve of degree $d \geq s+2$, which is either non-rational or non-smooth. Then $\operatorname{reg}(Y) \leq d-s+1$. 
Proof. This result follows again by consulting the list of all non-degenerate $(d-s+1)$-irregular curves in $\mathbf{P}^{s}$, which is given on page 504 of [G-L-P].

The next result is devoted to a further particular case.

(4.8) Proposition. Let $X \subseteq \mathbf{P}^{4}$ be a reduced, irreducible, non-degenerate surface of degree 6 which is $2-$ Buchsbaum. Then $\operatorname{reg}(X) \leq 5$.

Proof. Let $H=\mathbf{P}^{3} \subseteq \mathbf{P}^{4}$ be a generic hyperplane and let $Y=X \cap H$ be the corresponding hyperplane section, which is a reduced, irreducible, non-degenerate curve of degree 6 in $H=\mathbf{P}^{3}$. If $\operatorname{reg}(Y) \leq 4$, we may conclude by (4.5).

So, let $\operatorname{reg}(Y)>4$. Then, by (4.7), $Y$ is smooth and rational. So, $X$ satisfies our hypotheses (1.1) and (1.2) with $k=2$. In particular it follows from (1.6) that $Y$ is 2-Buchsbaum.

Now, let $h, l \in A_{1}$ be a generic pair of linear forms such that $H$ is defined by $h$. We may write $Y=\operatorname{Proj}(A / h A)$, and so (4.6) and (2.7) show that $h^{1}(A / h A)_{3}=1$. Writing $W:=H^{1}(A)_{3} / A_{1} H^{1}(A)_{2}$, applying cohomology to the sequence $0 \rightarrow A$ $(-1) \stackrel{h}{\rightarrow} A \rightarrow A / h A \rightarrow 0$ and observing that $h H^{1}(A)_{2} \subseteq A_{1} H^{1}(A)_{2}$ we get $\operatorname{dim}(W) \leq h^{1}(A / h A)_{3}=1$.

Next, let $(\lambda, \mu) \in K^{2}-\{(0,0)\}$. Then, we have epimorphisms $\lambda h+\mu l$ : $H^{1}(A)_{n} \rightarrow H^{1}(A)_{n+1} \rightarrow 0$ for all $n \geq 3$, (s. proof of (3.9)). Choosing $n=3$ and writing $\bar{h}$ resp. $\bar{l}$ for the maps $W \rightarrow H^{1}(A)_{4}$ induced by $h$ and $l$ (which exist as $\left.h A_{1} H^{1}(A)_{2}=l A_{1} H^{1}(A)_{2}=0\right)$, we thus get epimorphisms $\lambda \bar{h}+\mu \bar{l}: W \rightarrow H^{1}(A)_{4}$ $\rightarrow 0$ for all $(\lambda, \mu) \in K^{2}-\{(0,0)\}$. As $\operatorname{dim}(W) \leq 1$ we conclude that $H^{1}(A)_{4}=0$ (cf. $\left[\mathrm{Br}_{1},(3.2)\right]$ ) hence that $H^{1}(A)_{n}=0$ for all $n \geq 4$. In view of (2.7) B) and (1.5) c) this proves our claim.

To treat the general case, we need a last lemma.

(4.9) Lemma. Let $X \subseteq \mathbf{P}^{r}(r>3)$ be a reduced, irreducible non-degenerate surface which satisfies (1.1) and (1.2). Let $\alpha$ be defined as in (2.11). Then $\operatorname{reg}(X) \leq \alpha+2 k$.

Proof. By (1.5) and (3.12) we see that $h^{i}(A)_{n-t}=0$ for all $n \geq \beta+k$ and for $i=1,2,3$ where $\beta$ is defined according to (3.10). By (2.7) B) this shows that $\operatorname{reg}(X) \leq \beta+k$. As $\beta \leq \beta_{o} \leq \alpha+k$, this proves our claim. 
Now, we are ready to state and to prove the announced main result:

(4.10) Theorem. Let $X \subseteq \mathbf{P}^{r}(r>3)$ be a reduced, irreducible, non degenerate, 2-Buchsbaum surface of degree $d$. Then $\operatorname{reg}(X) \leq d-r+3$.

Proof. The cases $d \leq r$ are settled by [Ho-St-V, Thm. A]. So, let $d \geq r+1$. Let $H=\mathbf{P}^{r-1} \subseteq \mathbf{P}^{r}$ be a generic hyperplane and let $Y=X \cap H$. Then $Y$ is a reduced, irreducible and non-degenerate curve in $H=\mathbf{P}^{r-1}$, whose degree is $d \geq(r-1)+2$. If $Y$ is either non-rational or non-smooth, (4.7) shows that $\operatorname{reg}(Y) \leq d-(r-1)+1=d-r+2$. But then, (4.5) implies that $\operatorname{reg}(X) \leq d-r+2+1=d-r+3$. Hence, we may assume that $Y$ is smooth and rational, hence that $X$ satisfies the hypotheses (1.1) and (1.2) with $k=2$. If $d=r+1$, we conclude by (4.4).

So, let $d \geq r+2$. If $d=6$ and $r=4$, we may conclude by (4.8). So, let $(d, r) \neq(6,4)$. Then $d \geq r+\frac{r-1}{r-3}=\frac{r(r-2)-1}{r-3}$. This induces $d-\frac{d}{r-2}$ $=d \frac{r-3}{r-2} \geq \frac{r(r-2)-1}{r-2}=r-\frac{1}{r-2}$, thus $d-r \geq \frac{d-1}{r-2}$. As $d-r$ is an integer, this implies that $\alpha+1 \leq d-r$, where $\alpha$ is defined according to (2.11). So, by (4.9) we get $\operatorname{reg}(X) \leq d-r+3$.

\section{BIBLIOGRAPHY}

$\left[\mathrm{Br}_{1}\right]$ Brodmann, M., Bounds on the cohomological Hilbert functions of a projective variety, J. Algebra, 109 (1987), 352-380.

$\left[\mathrm{Br}_{2}\right] \quad$ - Sectional genus and cohomology of projective varieties, Math. Zeitschr., 208 (1991) $101-126$.

[E-Go] Eisenbud, D., Goto, S., Linear resolutions and minimal multiplicity, J. Algebra, 88 (1984), 89-133.

[Ev-Gr] Evans, E. G., Jr., Griffiths, P. A., Local cohomology modules for normal domains, J. London Math. Soc., 19 (1979), 277-284.

[F-V] Flenner, H., Vogel, W., Connectivity and its Applications to Improper Intersections in $\mathbf{P}^{n}$, Math. Gottingensis, Heft 53, Göttingen 1988.

[Fi-V] Fiorentini, M., Vogel, W., Old and New Results and Problems on Buchsbaum Modules, I*, Semin Geom., Univ. Studi Bologna 1988-1991, 53-61, Bologna 1991.

[Go] Goto, S., A Note on quasi-Buchsbaum Rings, Proc. Amer. Math. Soc., 90, (1984), 511-516.

[G-L-P] Gruson, L., Lazarsfeld, R,. Peskine, C., On a theorem of Castelnuovo and the equations defining space curves, Invent. Math., 72 (1983), 491-506.

[H] Harris, J., The genus of space curves, Math. Ann., 249 (1980), 191-204. 
[Ho-M-V] Hoa, T., Miro-Roig, R., Vogel,W., On numerical invariants of locally Cohen-Macaulay Schemes in $\mathbf{P}^{n}$, Preprint, MPI/0-57.

[Ho-St-V] Hoa, T., Stückrad, J., Vogel, W., Towards a structure theory for projective varieties of degree $=$ codimension +2 , J. Pure Appl. Algebra, 71 (1991), $203-231$.

[Ho-V] Hoa, T., Vogel, W., Castelnuovo-Mumford regularity and hyperplane sections, to appear in J. Algebra.

[L] Lazarsfeld, R., A sharp Castelnuovo bound for smooth surfaces, Duke Math. J., 55 (1987), 423-429.

$\left[\mathrm{Mu}_{1}\right] \quad$ Mumford, D., Pathologies III, Amer. J. Math. 89 (1967), 94-104.

$\left[\mathrm{Mu}_{2}\right]$ - Lectures on Curves on an Algebraic Surface, Annals of Math. Studies No 59, Princeton Univ. Press 1966.

[N] Nagel, U., Über Gradschranken für Syzygien and kohomologische Hilbertfunktionen, Diss. Univ. Paderborn, 1990.

$\left[\mathrm{St}-\mathrm{V}_{1}\right] \quad$ Stückrad, J., Vogel, W., Castelnuovo bounds for certain subvarieties of $\mathbf{P}^{n}$, Math. Ann., 276 (1987), 341-352.

$\left[\mathrm{St}-\mathrm{V}_{2}\right]-$ Castelnuovo's regularity and cohomological properties of sets of points in $\mathbf{P}^{n}$, Math. Ann., 284 (1989), 487-501.

M. Brodmann

Mathematisches Institut

der Universität

Rämistrasse 74

8001 Zürich, Switzerland

W. Vogel

Department of Mathematics

Massey University

Private Bag

Palmerston North, New Zealand 